\title{
Observation of a large, resonant, cross-Kerr nonlinearity in a cold Rydberg gas
}

\author{
Josiah Sinclair $\odot,{ }^{1, *}$ Daniela Angulo, ${ }^{1}$ Noah Lupu-Gladstein, ${ }^{1}$ Kent Bonsma-Fisher, ${ }^{1,2}$ and Aephraim M. Steinberg ${ }^{1,3}$ \\ ${ }^{1}$ Department of Physics and Centre for Quantum Information and Quantum Control, University of Toronto, \\ 60 St. George Street, Toronto, Ontario, Canada M5S $1 A 7$ \\ ${ }^{2}$ National Research Council of Canada, 100 Sussex Drive, Ottawa, Ontario, Canada K1A OR6 \\ ${ }^{3}$ Canadian Institute for Advanced Research, 180 Dundas Street West, Toronto, Ontario, Canada M5G $1 Z 8$
}

(Received 13 June 2019; published 20 December 2019)

\begin{abstract}
We report the experimental observation of a cross-Kerr nonlinearity in a free-space medium based on resonantly excited, interacting Rydberg atoms and electromagnetically induced transparency. The nonlinearity is used to implement cross-phase modulation between two optical pulses. The nonlinear phase written onto the probe pulse is measured to be as large as $8 \mathrm{mrad}$ per nanowatt of signal power, corresponding to a $\chi^{(3)}$ of $10^{-8} \mathrm{~m}^{2} / \mathrm{V}^{2}$. Potential applications range from optical quantum information processing to quantum nondemolition measurement of photon number.
\end{abstract}

DOI: 10.1103/PhysRevResearch.1.033193

\section{INTRODUCTION}

Fantastically strong interactions between high-lying Rydberg states combined with electromagnetically induced transparency (EIT) are an extremely promising platform for nonlinear optics at the level of single photons. In the last decade, Rydberg atoms have been used to demonstrate phase gates [1], photonic switches [2], atomic logic gates [3,4], quantum memory $[5,6]$, the generation of single-photon Fock states $[7,8]$, and other nonclassical states of light [9-12]. What these experiments share in common is that the nonlinear effects observed saturate near one photon. This is in contrast to a Kerr nonlinearity, where a medium experiences a linear shift in the index of refraction proportional to the number of photons in the medium. The Kerr effect has been studied extensively and has well-known applications in optical quantum computing $[13,14]$ and in generating and measuring nonclassical states of light $[15,16]$. In light of this, there have been several theoretical studies endeavoring to harness the powerful interactions of Rydberg atoms to implement a Kerr nonlinearity [17-23]. Noteworthy experimental results include the observation and study of a large dissipative (imaginary) $\chi^{(3)}$ [24-26] and the first observation of an off-resonant, dispersive, self-Kerr nonlinearity [26,27]. Notably, all experiments to date have involved a single beam, whereas most applications, such as photon number squeezing or quantum nondemolition measurement of photon number, require a cross-Kerr effect involving two beams.

We report an experimental observation of a dispersive cross-Kerr nonlinearity based on resonant Rydberg EIT. We

\footnotetext{
*sinclairjosiah@gmail.com

Published by the American Physical Society under the terms of the Creative Commons Attribution 4.0 International license. Further distribution of this work must maintain attribution to the author(s) and the published article's title, journal citation, and DOI.
}

observe that the phase shift acquired by a resonant optical pulse propagating through a cold cloud of atoms under EIT conditions depends linearly on the intensity of a second optical pulse. Our observations are consistent with a simple theoretical treatment based on van der Waals (vdW) interactions, which provides an intuitive explanation for the origin and scaling of the cross-Kerr nonlinearity.

\section{THEORY}

In our scheme, the signal pulse $\left[\Omega_{s}\right.$; see Fig. 1(a)] propagates on resonance under EIT conditions created by a resonant coupling beam $\left(\Omega_{c}\right)$. Inside the medium, signal photons excite Rydberg atoms, which interact via a vdW potential [28] of the form

$$
V(r)=-\hbar C_{6}\left(n^{*}\right) / r^{6},
$$

where $C_{6}\left(n^{*}\right)$ characterizes the strength of the vdW potential, $n^{*}$ is the adjusted principal quantum number [29], and $r$ is the distance between two Rydberg atoms. Due to the interactions, once an atom is excited to its Rydberg state, atoms in its vicinity will experience a position-dependent shift in their Rydberg levels, leading to a shift in the index of refraction [see Fig. 1(b)] seen by the probe $\left(\Omega_{p}\right)$. Assuming perfect EIT (100\% transparency on resonance and no dephasing) the per-atom phase shift acquired by the probe is

$$
\phi_{X}(r)=-\frac{\sigma}{2 \mathcal{A}} \times \frac{\Delta_{\mathrm{EIT}} V(r)}{\Delta_{\mathrm{EIT}}^{2}+V(r)^{2}},
$$

where $\Delta_{\text {EIT }}=\Omega_{c}^{2} / 2 \Gamma$ is the width of the EIT window and $\sigma /(4 \mathcal{A})$ is the theoretical peak per-atom phase shift given by the probe transition's resonant cross section $(\sigma)$ divided by the area $(\mathcal{A})$ of the probe focus [see the Appendix for a derivation of Eq. (2)]. From Eq. (2), we see that the phase shift acquired by the probe is maximized when $V(r)$ is comparable to $\Delta_{\mathrm{EIT}}$, and goes to zero in the limit of either $V(r) \rightarrow 0$ or $V(r) \rightarrow \infty$. Considering a cloud of atoms with a small number of Rydberg excitations distributed randomly, neither the subset of atoms 

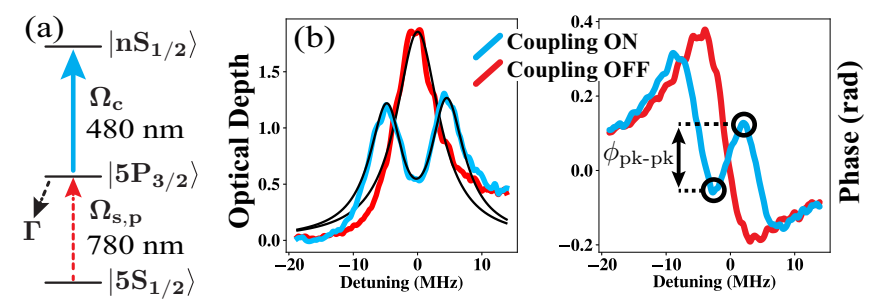

(c)

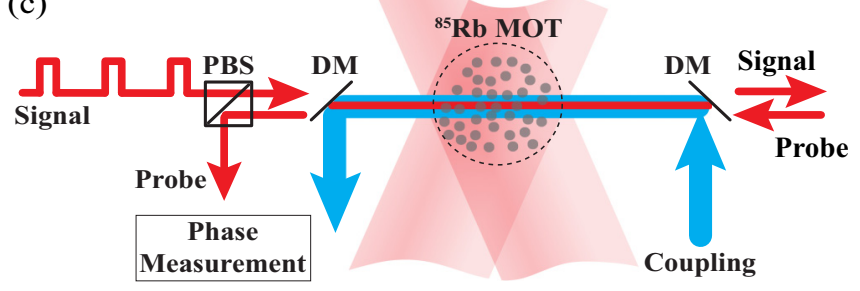

FIG. 1. (a) EIT level scheme. (b) A typical spectroscopy scan taken during the spectroscopy stage of our duty cycle $(n=49)$. From fit to spectra (black thin line) we extract experimental parameters such as EIT window width $\left(\Delta_{\text {EIT }}\right)$, peak OD with the coupling beam off, and $\phi_{\text {pk-pk }}$. (c) Experimental setup. Dichroic mirrors (DM) are used to overlap the probe and signal with the coupling beam on top of the ${ }^{85} \mathrm{Rb}$ magneto-optical trap (MOT). The probe and signal counterpropagate and are separated by polarizing beam splitters (PBS).

that are very far away from a Rydberg excitation nor the subset that are very close impart phase shifts to the probe. Instead, it is the small subset of atoms whose distance from a Rydberg excitation falls within a shell, such that their Rydberg levels are shifted by approximately $\Delta_{\mathrm{EIT}}$, which is responsible for shifting the phase of the probe. These are the atoms which are about $r_{b}=\left[C_{6} / \Delta_{\mathrm{EIT}}\right]^{1 / 6}$ away from a Rydberg excitation, where $r_{b}$ is the blockade radius [27,30,31]. The thickness of the shell, or rather, the range of distances where the interaction induces a shift comparable to $\Delta_{\text {EIT }}$ in the two-photon resonance, is determined (up to a numerical factor) by $r_{b}$, meaning that the fraction of atoms in the cloud that reside in this shell, and therefore impart a significant phase shift to the probe, is proportional to $r_{b}^{3}$ (and therefore to $\sqrt{C_{6} / \Delta_{\mathrm{EIT}}}$ ). It is notable that this scaling is identical to the one observed for off-resonant Rydberg nonlinearities [21,22,26,27], which are dominated by a different subset of atoms: those which reside within the volume $r_{b}^{3}$. In contrast, for our resonant Rydberg nonlinearity, the atoms which contribute reside in a shell near $r_{b}$ with atoms close to $r=0$ not contributing at all. The thickness of this shell is on the order of $r_{b}$, leading to the same scaling behavior as in previous work.

The total phase shift accumulated by the probe as it propagates through the medium can be found by summing up the contributions of each Rydberg excitation in the cloud,

$$
\left\langle\phi_{X}\right\rangle=\left(\rho_{\text {ryd }} \mathcal{A} L\right) \int_{0}^{\infty} \phi_{X}(r)\left(4 \pi r^{2} \rho\right) d r,
$$

where $\rho$ is the density of atoms, $\rho_{\text {ryd }}$ is the density of Rydberg excitations, and $L$ is the length of the medium [32]. The exact result is

$$
\left\langle\phi_{X}\right\rangle=-\frac{\pi}{4 \sqrt{2}} \times \mathrm{OD} \times\left[\frac{4}{3} \pi r_{b}^{3} \rho_{\mathrm{ryd}}\right],
$$

where optical depth $\mathrm{OD}=\rho \sigma L$. At low signal power, the Rydberg density is linear in atom density and in the power $\left(\mathcal{P}_{s} \propto\left|\Omega_{s}\right|^{2}\right)$ of the signal pulse:

$$
\rho_{\text {ryd }} \approx \rho\left|\Omega_{s}\right|^{2} /\left|\Omega_{c}\right|^{2} \text {. }
$$

Substituting this into Eq. (4), the total cross-phase shift is

$$
\left\langle\phi_{X}\right\rangle \approx-\frac{\mathrm{OD}}{2}\left[\frac{4}{3} \pi r_{b}^{3} \rho\right] \frac{\left|\Omega_{s}\right|^{2}}{\left|\Omega_{c}\right|^{2}} .
$$

Equation (6) is the main result of our theoretical treatment: the phase shift written on the probe is proportional to the optical depth of the interaction region, the power of the signal beam, and the number of Rydberg atoms in a blockade volume. A distinguishing property of this Kerr nonlinearity is its dependence on $r_{b}^{3}$, which in turn is proportional to $\sqrt{C_{6}\left(n^{*}\right)}$, making the phase proportional to $\left(n^{*}\right)^{5.5}$ when OD, $\Delta_{\mathrm{EIT}}, \Omega_{c}$ are held constant. This scaling is dramatically different from what would be expected from a nonlinearity due to the AC Stark shift, which has no Rydberg level dependence, or superradiant cascade decay, which scales as $\left(n^{*}\right)^{2.5}$ [33]. Additionally, our Kerr nonlinearity is cooperatively enhanced due to its quadratic dependence on atom density. This cooperative enhancement is reminiscent of a superatom model of Rydberg nonlinearity [34] and can be clearly seen in Eq. (6), where the Rydberg nonlinearity is that of a typical noninteracting atomic nonlinearity $\left(\left\langle\phi_{X}\right\rangle \sim-\mathrm{OD} \times I / I_{\text {sat }}\right)$ enhanced by the number of atoms in a blockade volume.

An important assumption made in the derivation of Eq. (6) is ideal EIT. In our experiment, we observed between 50\%$75 \%$ transparency on two-photon resonance [see Fig. 1(b)] for several reasons, including frequency instability of the lasers used, partial overlap of the probe and coupling beams, and broadening and dephasing caused by Rydberg-Rydberg interactions. We can relax this assumption by replacing OD/2 in Eq. (6) with a measured quantity $\phi_{\text {pk-pk }}$, which we extract from $\phi\left(\Delta_{p}\right)$, the EIT spectrum [see Fig. 1(b)]. The main remaining approximation under which Eq. (6) is valid is that $\rho_{\text {ryd }} \ll\left[\frac{4}{3} \pi r_{b}^{3}\right]^{-1}$. This guarantees that the Rydberg density remains linear in the signal power and ensures that nonlinear absorption due to Rydberg blockade can be neglected (loss is instead dominated by imperfect EIT). Maintaining a low Rydberg density requires low signal power $\left(\Omega_{c} \gg \Omega_{s}\right)$, but also low probe power $\left(\Omega_{c} \gg \Omega_{p}\right)$. This second constraint arises because probe photons can also excite atoms to the Rydberg state. Because we are only sensitive to cross-phase shifts, we cannot directly measure the presence of these background Rydberg atoms. However, they still interact via vdW forces, or decay to nearby $n P$ states and interact via stronger dipoledipole forces [35], resulting in degraded electromagnetically induced transparency, reduced $\phi_{\mathrm{pk} \text {-pk }}$, and smaller cross-phase shifts.

\section{EXPERIMENT AND RESULTS}

The experimental scheme is shown in Fig. 1(c). We prepare a gas of about $10^{7}{ }^{85} \mathrm{Rb}$ atoms in a magneto-optical trap (MOT) at a temperature of $60 \pm 10 \mu \mathrm{K}$. The MOT size is about $0.25 \mathrm{~mm}^{3}$ and the typical density is $\sim 4 \times$ $10^{10}$ atoms $/ \mathrm{cm}^{3}$. The atom duty cycle consists of $7.5 \mathrm{~ms}$ of MOT trapping, molasses, and free expansion, followed by 
a $1.2 \mathrm{~ms}$ measurement stage during which the phase and amplitude of the probe are measured via comparison to an off-resonant "reference" beam using beat-note interferometry [36]. The probe is at $780 \mathrm{~nm}$ and is resonant with $F=3$ to $F^{\prime}=4$. The reference is derived from the same master laser and is blue-detuned $2 \pi \times 100 \mathrm{MHz}$ from the same transition. The signal counterpropagates (see Fig. 1) with the probe and reference with orthogonal polarizations and is also resonant with $F=3$ to $F^{\prime}=4$. Setting the signal on resonance is particularly important in order to avoid the possibility of AC Stark shifts imparting an additional phase shift to the probe [37]. The typical resonant probe power is approximately 1 $\mathrm{nW}$ and the off-resonant "reference" probe is about $10 \mathrm{nW}$. The coupling power varies from $10-200 \mathrm{~mW}$ depending on the Rydberg level and the signal power is varied between $10 \mathrm{pW}$ to $100 \mathrm{nW}$. The probe and signal are focused to $20 \pm 4 \mu \mathrm{m}$ to ensure a constant waist over the length of the cloud $(L \approx 0.5 \mathrm{~mm})$. The coupling beam is locked on a $5 P \rightarrow n S$ resonance using EIT locking [31] and is focused to approximately $45 \mu \mathrm{m}$ in order to ensure a homogeneous coupling Rabi frequency across the transverse extent of the probe. The coupling Rabi frequency is typically $2 \pi \times(7 \pm 2) \mathrm{MHz}$, and the MOT OD is around 1-2. During the first $300 \mu$ s of the measurement stage, the transmission and phase of the probe are measured while the frequency is scanned across resonance three times (with no atoms, with atoms but no coupling beam, and finally with atoms and the coupling beam on). Figure 1(b) depicts the result of a frequency scan for $n=49$. The optical depth as a function of probe detuning, calculated from the transmission of the probe during the frequency scans, is fitted to theoretical absorption curves for two- and three-level atoms [thin black line in Fig. 1(b)] to extract the peak optical depth without EIT (OD), $\phi_{\mathrm{pk}-\mathrm{pk}}$, and the EIT window $\left(\Delta_{\mathrm{EIT}}\right)$. For the remaining $900 \mu \mathrm{s}$, the coupling and probe are left on and a signal pulse train consisting of 375 pulses, each $600 \mathrm{~ns}$ long and separated by $2.4 \mu \mathrm{s}$, is turned on. In order to isolate the effect of the signal pulse on the phase of the probe from other slow drifts, the phase is measured before, during, and after each signal pulse. This guarantees that we are only sensitive to cross-phase shifts.

The measured shift in the phase of the probe is plotted in Fig. 2 as a function of signal power for seven different $S$-state Rydberg levels (49, 54, 58, 62, 65, 68, and 70). The phase shift is linear up to $2-10 \mathrm{nW}$, depending on the Rydberg level, beyond which it saturates (data not shown). The slopes represent per-nanowatt cross-phase shifts and generally increase with Rydberg level, with the largest slope at $n=68$ (due to a larger OD used for this Rydberg level), where approximately $8 \mathrm{mrad}$ phase shifts were induced by $1 \mathrm{nW}$ of signal power. In order to compare our experiment with our theoretical treatment we use the following parameters: $1 \mathrm{nW} \rightarrow\left|\Omega_{s}\right|^{2} /\left|\Omega_{c}\right|^{2} \approx 0.01-0.02$, $4 \pi r_{b}^{3} \times \rho / 3 \approx 15-45$ for $n=68$, and $\phi_{\mathrm{pk}-\mathrm{pk}} \approx 0.05 \mathrm{rad}$. Substituting these into Eq. (6), we predict a cross-phase shift with a magnitude of $8-45 \mathrm{mrad} / \mathrm{nW}$, which is consistent with our results. Additionally, linear cross-phase modulation was observed across 2-3 orders of magnitude, persisting at signal powers as low as $\sim 20 \mathrm{pW}$ as shown in the inset in Fig. 2. Here $20 \mathrm{pW}$ corresponds to 47 photons in a $600 \mathrm{~ns}$ pulse, $<1$ photon in the medium at a time, based on our estimate of the interaction time, which we extract from the spectrum [Fig. 1(b)].

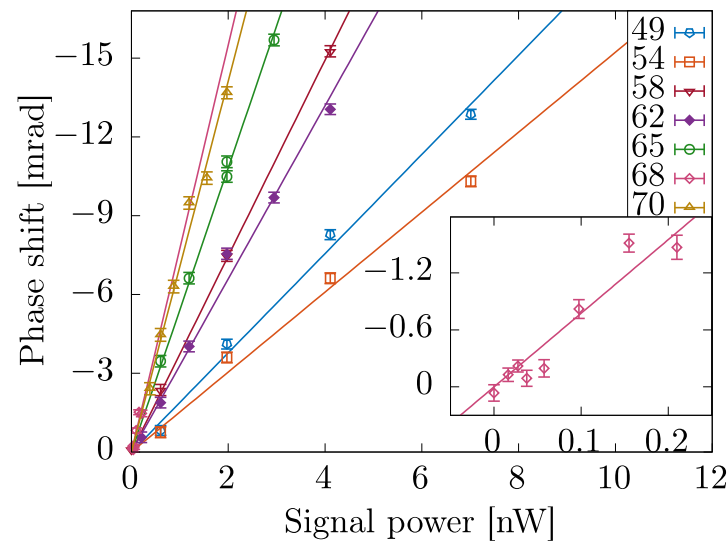

FIG. 2. The cross-phase shift acquired by the probe is plotted versus signal power for seven different Rydberg levels. In the inset, $n=68$ is isolated to show that the phase shift is measured down to powers as low as $\sim 20 \mathrm{pW}$. Lines of best fit are plotted on top of the data for each Rydberg level.

Next, we study the dependence of the cross-phase shift on probe power. We observe reduced electromagnetically induced transparency as we increase the probe power, a problem which is exacerbated for higher Rydberg levels. In Fig. 3, we vary the probe power and measure the cross-phase shift generated by $0.9 \mathrm{nW}$ of signal power for one of the higher Rydberg levels studied $(n=68)$. We observe a clear inverse relationship between the size of the cross-phase shift and the probe power which we attribute to background Rydberg excitations created by the probe beam rather than the signal beam. The number of Rydberg excitations in the interaction region grows with the total input photon number (signal and probe) and once $\rho_{\text {ryd }}$ approaches $\left[\frac{4}{3} \pi r_{b}^{3}\right]^{-1}$, the interaction region is effectively saturated. Because the medium is blockaded, the signal beam cannot create additional Rydberg excitations and does not induce any cross-phase shift on the probe. Based on our focus size $(\mathcal{A})$, saturation of the cross-phase shifts is expected to occur when the incident probe power reaches

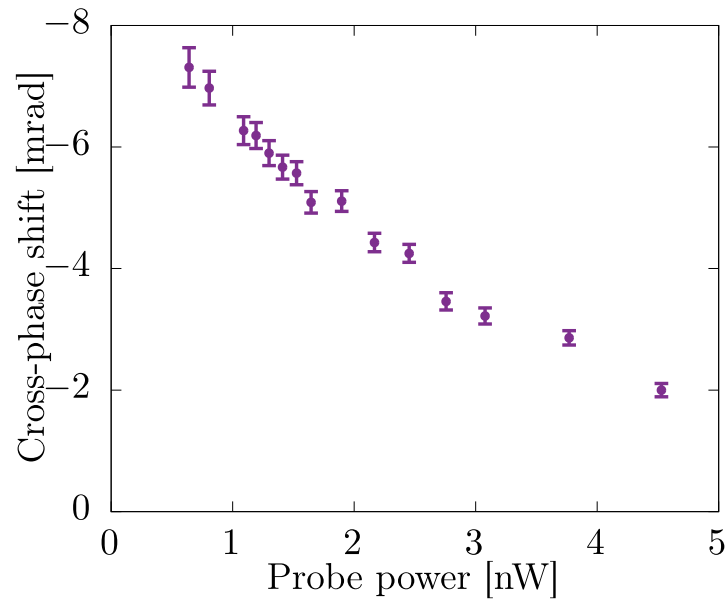

FIG. 3. The cross-phase shift acquired by the probe in the presence of $0.9 \mathrm{nW}$ of signal power is plotted as a function of onresonant probe power for $n=68$. The coupling Rabi frequency is $2 \pi \times(5.5 \pm 0.5) \mathrm{MHz}$. 


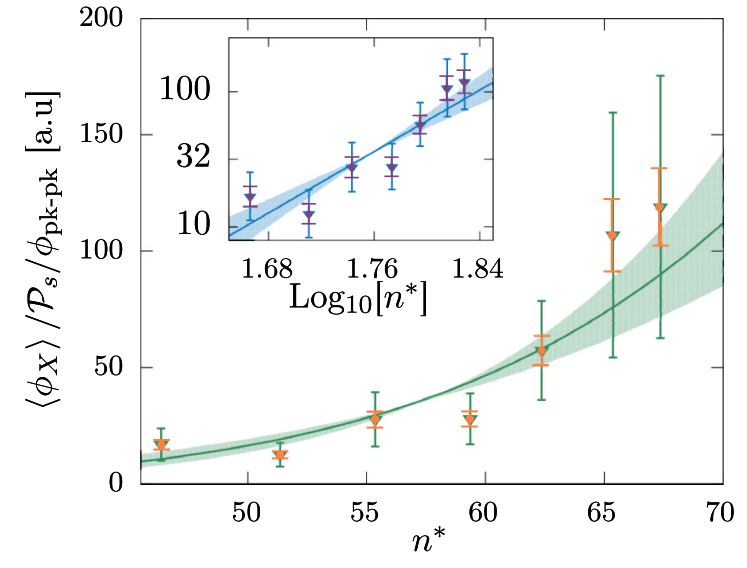

FIG. 4. $\left\langle\phi_{X}\right\rangle / \mathcal{P}_{s} / \bar{\phi}_{\mathrm{pk}-\mathrm{pk}}$ as a function of the adjusted Rydberg level. Here, $n^{*}=n-\delta$, where $\delta$ is the quantum defect and is approximately equal to 2.6 [29]. The original error bars are shown in orange (circles) and the revised (rescaled by $\sqrt{\chi^{(2)}}$ ) error bars in green (triangles). The inset shows the same data on a log scale. The data are fitted [green (blue) line] to a power law and the resulting fit has an exponent of $5.7 \pm 1.3$. The green (blue) shaded region indicates fits within one sigma of this estimate (4.4-7).

several nanowatts (when $\rho_{\text {ryd }}$ become comparable to $\left[\frac{4}{3} \pi r_{b}^{3}\right]^{-1}$ ), which is consistent with Fig. 3. In our experiment, self-phase modulation due to background probe-excited Rydberg atoms constrained the probe power to $1-2 \mathrm{nW}$ and limited the signal-to-noise ratio (lower probe powers were not used due to diminishing signal-to-noise). One possible way to mitigate self-phase effects in the future would be to address distinct Rydberg levels with the signal and probe and use a Förster resonance [38,39] to tune van der Waals interactions (self-phase) and dipole-dipole interactions (crossphase) separately.

We now turn to the Rydberg-level dependence of the cross-phase shift. The size of the Kerr nonlinearity, $\left\langle\phi_{X}\right\rangle / \mathcal{P}_{s}$, generally increased with Rydberg level. $\phi_{\text {pk-pk }}$ was also observed to vary strongly with Rydberg level, decreasing by roughly a factor of 2 between $n=49$ and $n=70$. This is likely due to background Rydberg atoms created by the probe, which saturate a fraction of the cloud that increases with $r_{b}^{3}$, contributing, among other things, to a smaller $\phi_{\mathrm{pk}-\mathrm{pk}}$. We fit lines to $\left\langle\phi_{X}\right\rangle / \mathrm{OD}$ versus signal power data for each Rydberg level. The fits, rescaled by $\phi_{\mathrm{pk}-\mathrm{pk}} / \overline{\mathrm{OD}}$ to eliminate spurious Rydberg-level dependence on $\phi_{\text {pk-pk }}$, are plotted as a function of Rydberg level in Fig. 4 (overbar indicates an average value over all trials for a given Rydberg level). The same data are inset on a log-log plot and fitted to a line. The error bars are calculated from the statistical uncertainty of the linear fit (ranging between 2\%-10\%), systemic uncertainty introduced by slow power drifts (estimated to be $10 \%$ ), and the uncertainty on $\phi_{\mathrm{pk}-\mathrm{pk}}$ (estimated to be 5\%-10\% based on noise in the measured spectra). The fit yields a power law with an exponent of 5.7, which is consistent with the predicted power law scaling, but the reduced $\chi^{2}$ is 11 .

We attribute this large $\chi^{2}$ to fluctuations of other experimental parameters not accounted for by $\phi_{\text {pk-pk }}$ between runs performed for different principal quantum numbers. For example, $\Delta_{\text {EIT }}$, whose impact on the cross-phase shifts is only
TABLE I. Comparison of selected Kerr nonlinearities $(*=$ hollow core fiber, $\star=$ not directly measured).

\begin{tabular}{lccc}
\hline \hline Description & $\chi^{(3)}\left(\mathrm{m}^{2} / \mathrm{V}^{2}\right)$ & $\left|\phi_{0}\right|(\mu \mathrm{rad} /$ photon $)$ & Ref. \\
\hline Fused silica & $2.5 \times 10^{-22}$ & & {$[40]$} \\
EIT, BEC & $5 \times 10^{-7}$ & & {$[42]$} \\
Rydberg, self-Kerr & $i \times\left(5 \times 10^{-7}\right)$ & & {$[25]$} \\
Rydberg, cross-Kerr & $1 \times 10^{-8}$ & $250^{\star}$ & This work \\
Rydberg, self-Kerr & $5 \times 10^{-9}$ & & {$[27]$} \\
N-scheme MOT & $2 \times 10^{-9}$ & 13 & {$[43]$} \\
N-scheme HCF* & $1 \times 10^{-12}$ & 300 & {$[41]$} \\
\hline \hline
\end{tabular}

partially accounted for by $\phi_{\mathrm{pk}-\mathrm{pk}}$, had a fractional variation of approximately $20 \%$ across all Rydberg levels. To take into account the high reduced $\chi^{2}$ and attempt to adequately estimate our uncertainties including the effects of these other fluctuations, we revise our estimate of the uncertainty of $\phi_{\mathrm{pk}-\mathrm{pk}}$ upward by a factor of $\sqrt{11}$, arriving at a fit parameter of $5.7 \pm 1.3$. This result leads us rule out the AC Stark shift and other non-Rydberg-based nonlinearities and to conclude that vdW-based interactions are the best explanation for the observed cross-Kerr nonlinearity.

\section{DISCUSSION}

Finally, we compare the size of our Rydberg-based crossKerr nonlinearity to those observed in other systems. The maximum $\chi^{(3)}$ observed was $\sim 1 \times 10^{-8} \mathrm{~m}^{2} / \mathrm{V}^{2}$ for $n=68$. This is much larger than the $\operatorname{Re}\left[\chi^{(3)}\right]$ available in conventional materials like fused silica [40], and similar in magnitude to the $\operatorname{Re}\left[\chi^{(3)}\right]$ reported in Ref. [27] (see Table I), in which Parigi et al. studied a cavity-enhanced self-Kerr effect based on offresonant Rydberg EIT. While larger nonlinearities have been observed in interacting Rydberg gases, for example [25], they were dissipative (the measured $\chi^{(3)}$ was imaginary), which makes them unsuitable for application to quantum state generation and quantum nondemolition measurement of photon number. Going beyond Rydberg-based Kerr nonlinearities, our $\operatorname{Re}\left[\chi^{(3)}\right]$ is 5000 times larger than the nonlinearity used by Venkataraman et al. [41] to observe cross-phase modulation in a hollow core fiber loaded with ultracold rubidium atoms. In fact, our $\operatorname{Re}\left[\chi^{(3)}\right]$ is only 50 times smaller than the record $\operatorname{Re}\left[\chi^{(3)}\right]$ observed in slow-light experiments at $\mathrm{BEC}$ densities despite the fact that our experiment was performed in a magneto-optical trap with 100 times lower density [42]. Finally, our measured $\operatorname{Re}\left[\chi^{(3)}\right]$ is 5 times larger than the $\operatorname{Re}\left[\chi^{(3)}\right]$ measured by Feizpour et al. based on EIT and AC Stark shifts (N-scheme) [43].

For many of the applications of strong single-photon-level nonlinearities a more important figure of merit than $\operatorname{Re}\left[\chi^{(3)}\right]$ is $\phi_{0}$, the phase shift per photon. Several of the experiments discussed here observed phase shifts per photon that ranged from $13 \mu \mathrm{rad} /$ photon [43] to $300 \mu \mathrm{rad} /$ photon [41]. Because our experiment was performed with long signal pulses, we did not directly measure the phase shift per signal photon. However, we did measure the spectrum and can therefore estimate the interaction time, allowing us to infer the average number of photons in the atomic medium at one time. In this 
way, we can indirectly estimate the per-photon phase shift to be $\sim-250 \mu \mathrm{rad} /$ photon.

In conclusion, we observed strong cross-phase modulation at low light levels generated by a resonant cross-Kerr nonlinearity based on Rydberg interactions and EIT. We directly measured cross-phase shifts and estimate the $\operatorname{Re}\left[\chi^{(3)}\right]$ to be $10^{-8} \mathrm{~m}^{2} / \mathrm{V}^{2}$. We varied the Rydberg level and observed a scaling with the principal quantum number consistent with the $\left(n^{*}\right)^{5.5}$ expected for a vdW-based nonlinearity. This is an experimental demonstration of a cross-Kerr nonlinearity between two beams based on Rydberg interactions. Future experiments will explore ways to reduce self-phase interactions, which, when combined with higher density and higher Rydberg levels, should enable even larger single-photon level dispersive nonlinearities with important applications in quantum optics and quantum information.

\section{ACKNOWLEDGMENTS}

This research was supported by NSERC, CIFAR, and the Fetzer Franklin Fund of the John E. Fetzer Memorial Trust. A.M.S. is a fellow in the CIFAR Quantum Information Science program. The authors are grateful to Hudson Pimenta for valuable discussions, and Alan Stummer for equipment design.

\section{APPENDIX: DERIVATION OF THE PER-ATOM PHASE SHIFT}

We start by considering an atom illuminated by two laser beams denoted with the subscript $p$ for probe and $c$ for coupling in a ladder configuration [see Fig. 1(a)]. The standard result for the steady state coherence $\left(\rho_{e g}\right)$ for a three-level atom in a rotating wave frame and under a rotating wave approximation is [44]

$$
\rho_{e g}=\frac{i \Omega_{p}}{2\left(\frac{\Gamma}{2}-i \Delta_{p}+\frac{\Omega_{c}^{2}}{4\left[\gamma-i\left(\Delta_{c}+\Delta_{p}\right)\right]}\right)} .
$$

Here, $\Gamma$ is the natural linewidth of the intermediate state $(|e\rangle)$, $\Delta_{p}$ is the detuning of the probe beam from resonance, $\Delta_{c}$ is the detuning of the coupling beam from resonance, $\Omega_{c}$ is the Rabi frequency of the coupling beam, $\Omega_{p}$ is the Rabi frequency of the probe beam, and $\gamma$ is the natural linewidth of the Rydberg state $(|r\rangle)$. For simplicity we start by setting $\gamma=0$ and $\Delta_{p}=0$. This is equivalent to assuming that the probe is always on single-photon resonance, and that the Rydberg lifetime is much longer than the timescales we are interested in. Under these assumptions $\rho_{e g}$ simplifies to

$$
\rho_{e g}=\frac{i \Omega_{p} \Delta_{c} / \Gamma}{\left(\Delta_{c}+i \Delta_{\mathrm{EIT}}\right)},
$$

where $\Delta_{\mathrm{EIT}}=\Omega_{c}^{2} /(2 \Gamma)$ is the width of the transparency window. The phase shift a cloud of atoms imparts to the probe laser as it propagates through is

$$
\phi=\operatorname{Re}[\Delta n] k L,
$$

where $\Delta n$ is the change in the index of refraction due to the atoms being present, $k$ is the wave number of the probe, and $L$ is the length of the medium. The change in the index of refraction due to the atoms is the electric susceptibility, $\Delta n=\chi$, which is related to the steady state coherence,

$$
\epsilon_{0} \chi\langle E\rangle=N e\langle\hat{x}\rangle=N e x_{0} \rho_{e g},
$$

where $\epsilon_{0}$ is the vacuum permittivity, $N$ is the atomic density, $e$ is the charge of an electron, $x_{0}$ is the transition matrix dipole element, and $\langle E\rangle$ is the time-averaged electric field amplitude of the probe, related to the probe Rabi frequency,

$$
\Omega_{p}=\frac{e x_{0}\langle E\rangle}{\hbar} .
$$

Substituting $\Omega_{p}$ for $\langle E\rangle$, the electric susceptibility is

$$
\chi=N \frac{e^{2} x_{0}^{2}}{\epsilon_{0} \hbar} \frac{\rho_{e g}}{\Omega_{p}},
$$

and the phase shift imparted to the probe is

$$
\phi=N L \frac{e^{2} x_{0}^{2} \omega}{\epsilon_{0} \hbar c} \frac{\operatorname{Re}\left[\rho_{e g}\right]}{\Omega_{p}},
$$

where $k$ has been substituted for $\omega / c$. The atomic cross section $\sigma$ is defined such that

$$
\frac{\Gamma}{2} \sigma=\frac{e^{2} x_{0}^{2} \omega}{\epsilon_{0} \hbar c} .
$$

We can substitute Eq. (A2) into Eq. (A1) to simplify the phase shift (substituting OD $=N \sigma L$ ),

$$
\phi=\mathrm{OD} \frac{\Gamma / 2}{\Omega_{p}} \operatorname{Re}\left[\rho_{e g}\right] .
$$

Taking the real part of $\rho_{e g}$ and substituting into Eq. (A3), the phase shift acquired by the probe is

$$
\phi=\frac{\mathrm{OD}}{2} \frac{\Delta_{\mathrm{EIT}} \Delta_{c}}{\Delta_{c}^{2}+\Delta_{\text {EIT }}^{2}} .
$$

We define an average "per-atom" contribution, $\sigma / \mathcal{A}$, by dividing the OD by $\mathcal{N}$, the number of atoms,

$$
\frac{\mathrm{OD}}{\mathcal{N}}=\frac{N \sigma L}{\mathcal{N}}=\frac{\mathcal{N} \sigma L}{\mathcal{N} V}=\frac{\sigma}{\mathcal{A}},
$$

where $V=\mathcal{A} L$ is the volume of the interaction region, $\mathcal{A}$ is the area of the probe waist, and $L$ is the length of the interaction region. The per-atom phase shift imparted to the probe by the atoms is therefore

$$
\phi=\frac{\sigma}{2 \mathcal{A}} \frac{\Delta_{\mathrm{EIT}} \Delta_{c}}{\Delta_{c}^{2}+\Delta_{\text {EIT }}^{2}},
$$

which is the result used in Eq. (2) in the main body of the paper, where the coupling beam is assumed to be on resonance and so any detuning from the Rydberg level is due to van der Waals interactions with a nearby Rydberg excitation $\left[\Delta_{c}=\right.$ $V(r)]$. 
[1] D. Tiarks, S. Schmidt-Eberle, T. Stolz, G. Rempe, and S. Dürr, A photon-photon quantum gate based on Rydberg interactions, Nat. Phys. 15, 124 (2019).

[2] S. Baur, D. Tiarks, G. Rempe, and S. Dürr, Single-Photon Switch Based on Rydberg Blockade, Phys. Rev. Lett. 112, 073901 (2014).

[3] M. Müller, I. Lesanovsky, H. Weimer, H. P. Büchler, and P. Zoller, Mesoscopic Rydberg Gate Based on Electromagnetically Induced Transparency, Phys. Rev. Lett. 102, 170502 (2009).

[4] T. Keating, R. L. Cook, A. M. Hankin, Y.-Y. Jau, G. W. Biedermann, and I. H. Deutsch, Robust quantum logic in neutral atoms via adiabatic Rydberg dressing, Phys. Rev. A 91, 012337 (2015).

[5] E. Distante, A. Padrón-Brito, M. Cristiani, D. Paredes-Barato, and H. de Riedmatten, Storage Enhanced Nonlinearities in a Cold Atomic Rydberg Ensemble, Phys. Rev. Lett. 117, 113001 (2016).

[6] D. Maxwell, D. J. Szwer, D. Paredes-Barato, H. Busche, J. D. Pritchard, A. Gauguet, K. J. Weatherill, M. P. A. Jones, and C. S. Adams, Storage and Control of Optical Photons Using Rydberg Polaritons, Phys. Rev. Lett. 110, 103001 (2013).

[7] Y. O. Dudin and A. Kuzmich, Strongly interacting Rydberg excitations of a cold atomic gas, Science 336, 887 (2012).

[8] F. Ripka, H. Kübler, R. Löw, and T. Pfau, A room-temperature single-photon source based on strongly interacting Rydberg atoms, Science 362, 446 (2018).

[9] O. Firstenberg, T. Peyronel, Q.-Y. Liang, A. V. Gorshkov, M. D. Lukin, and V. Vuletic, Attractive photons in a quantum nonlinear medium, Nature (London) 502, 71 (2013).

[10] T. Peyronel, O. Firstenberg, Q.-Y. Liang, S. Hofferberth, A. V. Gorshkov, T. Pohl, M. D. Lukin, and V. Vuletic, Quantum nonlinear optics with single photons enabled by strongly interacting atoms, Nature (London) 488, 57 (2012).

[11] J. D. Thompson, T. L. Nicholson, Q.-Y. Liang, S. H. Cantu, A. V. Venkatramani, S. Choi, I. A. Fedorov, D. Viscor, T. Pohl, M. D. Lukin, and V. Vuletic, Symmetry-protected collisions between strongly interacting photons, Nature (London) 542, 206 (2017).

[12] Q.-Y. Liang, A. V. Venkatramani, S. H. Cantu, T. L. Nicholson, M. J. Gullans, A. V. Gorshkov, J. D. Thompson, C. Chin, M. D. Lukin, and V. Vuletić, Observation of three-photon bound states in a quantum nonlinear medium, Science 359, 783 (2018).

[13] K. Nemoto and W. J. Munro, Nearly Deterministic Linear Optical Controlled-NOT Gate, Phys. Rev. Lett. 93, 250502 (2004).

[14] W. J. Munro, K. Nemoto, and T. P. Spiller, Weak nonlinearities: A new route to optical quantum computation, New J. Phys. 7, 137 (2005).

[15] N. Imoto, H. A. Haus, and Y. Yamamoto, Quantum nondemolition measurement of the photon number via the optical Kerr effect, Phys. Rev. A 32, 2287 (1985).

[16] D. Schmid, K. Marshall, and D. F. V. James, Verifying crossKerr induced number squeezing: A case study, J. Mod. Opt. 64, 2306 (2017).

[17] Z. Bai and G. Huang, Enhanced third-order and fifth-order Kerr nonlinearities in a cold atomic system via Rydberg-Rydberg interaction, Opt. Express 24, 4442 (2016).
[18] C. Ates, S. Sevinçli, and T. Pohl, Electromagnetically induced transparency in strongly interacting Rydberg gases, Phys. Rev. A 83, 041802(R) (2011).

[19] S. Sevinçli, N. Henkel, C. Ates, and T. Pohl, Nonlocal Nonlinear Optics in Cold Rydberg Gases, Phys. Rev. Lett. 107, 153001 (2011).

[20] J. Stanojevic, V. Parigi, E. Bimbard, A. Ourjoumtsev, and P. Grangier, Dispersive optical nonlinearities in a Rydberg electromagnetically-induced-transparency medium, Phys. Rev. A 88, 053845 (2013).

[21] A. Grankin, E. Brion, E. Bimbard, R. Boddeda, I. Usmani, A. Ourjoumtsev, and P. Grangier, Quantum statistics of light transmitted through an intracavity Rydberg medium, New J. Phys. 16, 043020 (2014).

[22] A. Grankin, E. Brion, E. Bimbard, R. Boddeda, I. Usmani, A. Ourjoumtsev, and P. Grangier, Quantum-optical nonlinearities induced by Rydberg-Rydberg interactions: A perturbative approach, Phys. Rev. A 92, 043841 (2015).

[23] P. Bienias and H. P. Büchler, Quantum theory of Kerr nonlinearity with Rydberg slow light polaritons, New J. Phys. 18, 123026 (2016).

[24] J. D. Pritchard, D. Maxwell, A. Gauguet, K. J. Weatherill, M. P. A. Jones, and C. S. Adams, Cooperative Atom-Light Interaction in a Blockaded Rydberg Ensemble, Phys. Rev. Lett. 105, 193603 (2010).

[25] J. D. Pritchard, A. Gauguet, K. J. Weatherill, and C. S. Adams, Optical non-linearity in a dynamical Rydberg gas, J. Phys. B: At., Mol. Opt. Phys. 44, 184019 (2011).

[26] R. Boddeda, I. Usmani, E. Bimbard, A. Grankin, A. Ourjoumtsev, E. Brion, and P. Grangier, Rydberg-induced optical nonlinearities from a cold atomic ensemble trapped inside a cavity, J. Phys. B: At., Mol. Opt. Phys. 49, 084005 (2016).

[27] V. Parigi, E. Bimbard, J. Stanojevic, A. J. Hilliard, F. Nogrette, R. Tualle-Brouri, A. Ourjoumtsev, and P. Grangier, Observation and Measurement of Interaction-Induced Dispersive Optical Nonlinearities in an Ensemble of Cold Rydberg Atoms, Phys. Rev. Lett. 109, 233602 (2012).

[28] K. Singer, J. Stanojevic, M. Weidemüller, and R. Côté, Longrange interactions between alkali Rydberg atom pairs correlated to the $n \mathrm{~s}-n \mathrm{~s}, n \mathrm{p}-n \mathrm{p}$, and $n \mathrm{~d}-n \mathrm{~d}$ asymptotes, J. Phys. B: At., Mol. Opt. Phys. 38, S295 (2005).

[29] W. Li, I. Mourachko, M. W. Noel, and T. F. Gallagher, Millimeter-wave spectroscopy of cold Rb Rydberg atoms in a magneto-optical trap: Quantum defects of the $n s, n p$, and $n d$ series, Phys. Rev. A 67, 052502 (2003).

[30] A. Gaëtan, Y. Miroshnychenko, T. Wilk, A. Chotia, M. Viteau, D. Comparat, P. Pillet, A. Browaeys, and P. Grangier, Observation of collective excitation of two individual atoms in the Rydberg blockade regime, Nat. Phys. 5, 115 (2009).

[31] R. P. Abel, A. K. Mohapatra, M. G. Bason, J. D. Pritchard, K. J. Weatherill, U. Raitzsch, and C. S. Adams, Laser frequency stabilization to excited state transitions using electromagnetically induced transparency in a cascade system, Appl. Phys. Lett. 94, 071107 (2009).

[32] For simplicity we have assumed that the probability of Rydberg excitations is low enough to neglect any spatial correlations in the Rydberg density. 
[33] J. D. Pritchard, Cooperative Optical Non-Linearity in a Blockaded Rydberg Ensemble, Ph.D. thesis, Durham University, 2011.

[34] T. M. Weber, M. Höning, T. Niederprüm, T. Manthey, O. Thomas, V. Guarrera, M. Fleischhauer, G. Barontini, and H. Ott, Mesoscopic Rydberg-blockaded ensembles in the superatom regime and beyond, Nat. Phys. 11, 157 (2015).

[35] T. Boulier, E. Magnan, C. Bracamontes, J. Maslek, E. A. Goldschmidt, J. T. Young, A. V. Gorshkov, S. L. Rolston, and J. V. Porto, Spontaneous avalanche dephasing in large Rydberg ensembles, Phys. Rev. A 96, 053409 (2017).

[36] G. Dmochowski, 8-Port Homodyne Detection of EIT-Enhanced Cross-Phase Modulation Using Broadband Signal Pulses, Ph.D. thesis, University of Toronto, 2016.

[37] A. Feizpour, G. Dmochowski, and A. M. Steinberg, Short-pulse cross-phase modulation in an electromagnetically-inducedtransparency medium, Phys. Rev. A 93, 013834 (2016).

[38] I. I. Ryabtsev, D. B. Tretyakov, I. I. Beterov, and V. M. Entin, Observation of the Stark-Tuned Förster Resonance between Two Rydberg Atoms, Phys. Rev. Lett. 104, 073003 (2010).

[39] D. B. Tretyakov, V. M. Entin, E. A. Yakshina, I. I. Beterov, C. Andreeva, and I. I. Ryabtsev, Controlling the interactions of a few cold Rb Rydberg atoms by radio-frequency-assisted Förster resonances, Phys. Rev. A 90, 041403(R) (2014).

[40] R. W. Boyd, Nonlinear Optics, 3rd ed. (Academic Press, Inc., Orlando, FL, USA, 2008).

[41] V. Venkataraman, K. Saha, and A. L. Gaeta, Phase modulation at the few-photon level for weak-nonlinearity-based quantum computing, Nat. Photonics 7, 138 (2013).

[42] L. V. Hau, S. E. Harris, Z. Dutton, and C. H. Behroozi, Light speed reduction to 17 metres per second in an ultracold atomic gas, Nature (London) 397, 594 (1999).

[43] A. Feizpour, M. Hallaji, G. Dmochowski, and A. M. Steinberg, Observation of the nonlinear phase shift due to single postselected photons, Nat. Phys. 11, 905 (2015).

[44] J. Pritchard, Cooperative Optical Non-Linearity in a Blockaded Rydberg Ensemble (Springer, 2012). 